\title{
Guía didáctica de apoyo al Autoexamen Bucal
}

\author{
Teaching guide to support the Oral Self Exam
}

\author{
Dra. María Isabel Garay Crespo. ${ }^{1}$ Est. Jessica Rubiera Carballosa. ${ }^{2}$ Est. Viviana González
} Escolarte. ${ }^{3}$ \& Est. Maricarmen Rodríguez Domínguez. ${ }^{4}$

Recibido: 14-01-2020 / Revisado: 16-02-2020 / Aceptado: 18-03-2020 / Publicado: 03-04-2020

\section{DOI: https://doi.org/10.33262/anatomiadigital.v3i2.1188}

\begin{abstract}
.
Oral self-examination is one of the preventive methods to detect oral cancer.

Objective: To implement a Didactic Guide to support Oral Self-Examination for use in preventive activities of oral cancer.

Methodological design: An investigation of technological innovation was carried out with experimental design, where, based on the identification of learning needs regarding oral self-examination, a teaching medium was designed to support this procedure, validated by experts and began to be applied in December 2018. 47 people over 60 years old belonging to the Grandparents House
\end{abstract}

\section{Resumen.}

El autoexamen bucal es uno de los métodos preventivos para detectar el cáncer bucal. Objetivo: Implementar una Guía didáctica de apoyo al Autoexamen Bucal para su uso en las actividades preventivas del cáncer bucal. Diseño metodológico: Se realizó una investigación de innovación tecnológica con diseño experimental, donde a partir de la identificación de las necesidades de aprendizaje con respecto al autoexamen bucal se diseñó un medio de enseñanza de apoyo a este procedimiento, validado por expertos y se comenzó a aplicar en diciembre del 2018. Se toma como universo 47 personas mayores de 60 años pertenecientes a la Casa

\footnotetext{
${ }^{1}$ Universidad de Ciencias Médicas de Matanzas. Matanzas, Cuba, Especialista de 1er grado en Estomatología General Integral, garaymmtz@infomed.sld.cu iD https://orcid.org/0000-0002-7353-0236

2 Universidad de Ciencias Médicas de Matanzas. Matanzas, Cuba, Estudiante tercer año Estomatología, jrubieramtz@infomed.sld.cu iD https://orcid.org/0000-0002-7353-0237

3 Universidad de Ciencias Médicas de Matanzas. Matanzas, Cuba, Estudiante tercer año Estomatología, vgonzalezmtz@infomed.sld.cu iD https://orcid.org/0000-0002-7353-0238

${ }^{4}$ Universidad de Ciencias Médicas de Matanzas. Matanzas, Cuba, Estudiante tercer año Estomatología, mrodriguezmtz@infomed.sld.cu iD https://orcid.org/0000-0002-7353-0239
} 
“Antonio Dicirst Martínez” of Matanzas are taken as universe.

Results: $100 \%$ of respondents were unaware of oral self-examination and $89.36 \%$ indicated interest in learning about oral health. $70.21 \%$ of the total did not present smoking habits and with respect to alcohol consumption, $80.25 \%$ said they did not drink alcoholic beverages. $87.23 \%$ of respondents considered the implementation of educational material as positive, without any negative criteria.

Conclusions: All respondents were unaware of oral self-examination and expressed interest in learning about this topic. A Guide to support oral self-examination was developed and its implementation was subsequently evaluated where the level of satisfaction of the participants in this research was demonstrated.

Keywords: oral self-examination, teaching medium, Oral Cancer Detection Program and oral cancer. de Abuelos “Antonio Dicirst Martínez" de Matanzas.

Resultados: El $100 \%$ de los encuestados desconocían sobre el autoexamen bucal y el $89.36 \%$ señaló interés por aprender sobre salud bucal. El 70.21\% del total no presentó hábito de fumar y con respecto al consumo de alcohol el $80.25 \%$ manifestó la no ingestión de bebidas alcohólicas. El $87.23 \%$ de los encuestados consideró como positivo la implementación del material educativo, sin ningún criterio negativo.

Conclusiones: La totalidad de los encuestados desconocían sobre autoexamen bucal yrefirió interés para aprender acerca de este tema. Se elaboró una Guía de apoyo al Autoexamen bucal y posteriormente se evaluó su implementación donde quedó demostrado el nivel de satisfacción de los participantes en esta investigación.

Palabras claves: autoexamen bucal, medio de enseñanza, Programa de Detección del Cáncer Bucal y cáncer bucal.

\section{Introducción.}

El cáncer constituye uno de los mayores problemas que enfrenta la humanidad no solo para la salud pública sino para las demás ciencias en general. Es un término común para designar a todos los tumores malignos, que deriva de la palabra latina canoer (cangrejo), siendo Hipócrates (469 a 370 ac) quien lo describió por primera vez, utilizando los términos carcinos y carcinomas, otros autores lo han definido como un proceso tumoral que ocurre en todas las poblaciones humanas y animales, apareciendo en los tejidos compuestos por células con potencialidad de división. (Calunga\&Castillo A., 2014)

Como entidad maligna tiene un gran índice de mortalidad, considerándose el hecho de que cada año mueren en el mundo cuatro millones de personas por este padecimiento, de mantenerse dicha tendencia será considerada la primera causa de muerte en este siglo. (Celedón, 2012) 
El estudio de esta enfermedad se ha enmarcado en tres periodos importantes, el primero fue descriptivo y se extendió desde los principios de la historia hasta mediados del siglo XVIII; el segundo, profundizó en los aspectos clínicos y anatomopatológicos, y culminó cerca del año 1900; mientras que el tercer período se extiende hasta la época actual e incluye la definición y el diagnóstico molecular, así como los avances terapéuticos. (Celedón, 2012)

En el siglo $\mathrm{XX}$, las investigaciones y descubrimientos relacionados con el cáncer experimentaron avances muy significativos debido a la prevalencia y la incidencia progresiva de esta enfermedad; pero no fue hasta el año 1964, que se reforzó la importancia de los factores de riesgo de los tumores malignos, luego de aparecer un documento del gobierno norteamericano denominado Smoking and Health. United States. Report of the Advisory Committee to the Surgical General of the Public Health Service. (Fumar y Salud. Reporte del Comité Asesor para la Cirugía General del Servicio de Salud Pública de los Estados Unidos).(J., 2007) (Guerrero Brito, Pérez Cabrera, \& Hernández Abreu, 2020)

En este artículo, se mostró la relación directa del tabaco con varios tipos de cáncer, lo que causó una reacción de miedo o terror en todo el mundo, pues por primera vez, una institución gubernamental de alto nivel, con estupenda notoriedad científica, oficializó la correspondencia entre el tabaquismo y varias enfermedades fatales.(Guerrero Brito, Pérez Cabrera, \& Hernández Abreu, 2020) (ID., Kalpidis , Chadiipantelis , \& Konstantinidis , 2009)

Se prevé que en el período comprendido de 2007 al 2030 la mortalidad por cáncer se incremente entre 7 y 17 millones a nivel mundial, debido en parte al crecimiento demográfico y al envejecimiento de la población (Organization, 2008).La organización Mundial de la Salud (OMS), calcula que en el 2005 murieron de cáncer 7,6 millones de personas y que en los próximos 10 años morirán 84 millones más si no se emprenden acciones (Salud, 2012)

La cavidad bucal, por su situación anatómica especial, sus múltiples funciones, así como su exposición permanente a agentes físicos, químicos y biológicos, merece una cuidadosa atención médica tanto en la prevención, como en la detección precoz de cualquier afección.(Ramos Francisco \& Calero Barreto, 2019)

Se ha podido comprobar, por investigaciones realizadas en países industrializados occidentales, que el cáncer de la mucosa bucal representa el octavo tumor más frecuente y los índices epidemiológicos indican una tendencia al incremento progresivo. Incluye las lesiones malignas del labio, la cavidad bucal y la bucofaringe; es más frecuente en hombres, con mayor incidencia después de los 40 años, sin distinción de área geográfica. (J, González, \& González García, 2019)

Existen factores de riesgo como el tabaquismo, el alcoholismo, la sepsis bucal, los dientes con bordes filosos, las prótesis dentales desajustadas, la exposición excesiva la radiación solar, la dieta escasa en frutas y vegetales, la acción de virus, hongos, oncogenes y otros 
factores generales. La combinación de dos o más factores aumenta la probabilidad de padecer la enfermedad. El adecuado nivel de conocimientos acerca de los factores que producen el cáncer bucal, contribuye a su prevención.(Calunga \& Castillo A., 2014)

En Cuba, el cáncer bucal se mantiene entre las diez primeras localizaciones de tumores desde el año 1970, ocupa entre el séptimo y décimo lugares. Al comparar el año 2011 con 1970, la tasa de mortalidad ha sufrido un incremento del $23,8 \%$, a razón de tres hombres por cada mujer.(C, 2011)(Pública, 2009)La lucha contra el cáncer debe ser interdisciplinaria y debe encaminarse, sobre todo, hacia la prevención en tres fases o niveles:

- La prevención primaria que es la que se realiza para disminuir la probabilidad de que se produzca la enfermedad y se dice ser la más importante.

- La prevención secundaria pasa por la detección y terapéuticas tempranas de la lesión preneoplásica o cancerosa, lo que conlleva a un diagnóstico y tratamiento precoz, que permitirá curarla con mayor facilidad.

- La prevención terciaria que consiste en actuar sobre la enfermedad cancerosa cuando ya está bien establecida, con secuelas o sin ellas y los tratamientos realizados en esta fase de la prevención son muy agresivos y sería deseable no llegar a estas situaciones, por lo que debemos potenciar las medidas preventivas en sus fases primaria y secundaria, para una mejor rehabilitación del paciente.(Rodríguez Ricardo E., y otros, 2014)(Espinosa Ortiz, 2019) (GL \& Miranda Tarragó, 2009)

En 1982 comenzó en Cuba la aplicación del Programa de Detección Precoz del Cáncer Bucal, único en el mundo por su cobertura nacional. Se basa en un examen minucioso del complejo bucomaxilofacial con una periodicidad anual en los individuos mayores de 15 años.(ND., Rodríguez, \& García JM, 2009)

En el Programa de Detección del Cáncer Bucal (PDCB) se orienta a todas las personas que acuden a los servicios e instituciones de salud sobre la prevención de esa enfermedad y realiza la promoción a través de los diferentes medios de difusión. Uno de los objetivos de este programa está orientado a disminuir la morbilidad y mortalidad por cáncer bucal. (I., Suarez Zafra, Leyva Labrador, Rodríguez, \& Navarro Espinosa, 2009)

En sus inicios, este programa utilizó a los estomatólogos para realizar el examen bucal y propuso que todos los sujetos de 15 y más años recibieran anualmente un estudio de la boca y el cuello en el momento de acudir a la consulta estomatológica, pero la cobertura completa no se ha alcanzado debido probablemente a que la población supuestamente sana no demanda el chequeo periódico de su boca y dentro de los objetivos de este programa se contempló desarrollar actividades de promoción y educación para la salud, diagnóstico temprano, rehabilitación y seguimiento evolutivo de los casos, capacitación del personal, así como la 
actividad investigativa de controlar y evaluar estas acciones en los servicios estomatológicos.(C, 2011)

Entre las medidas preventivas que incluye este programa se encuentra la realización del Auto examen Bucal de forma sistemática y correcta. A pesar de lo referido se ha venido constatando en los últimos años que los resultados alcanzados con la aplicación de este programa, incluyendo el Auto examen Bucal, no han sido los esperados, se evidencian dificultades(ND., Rodríguez, \& García JM, 2009).

Es evidente la importancia que reviste contemplar esta situación, y tener presente que las medidas preventivas y de promoción de salud constituyen valiosas herramientas para la detección precoz de estas lesiones, cuyo propósito está dirigido al paciente, a la familia y a su entorno, donde estomatólogos y médicos se muestren más activos y que comprometa a otras especialidades del segundo nivel en aras de un diagnóstico precoz y la rehabilitación integral de quienes padecen esta enfermedad.(I., Suarez Zafra, Leyva Labrador, Rodríguez, \& Navarro Espinosa, 2009)

Debemos tener presente que el arte de curar y aliviar dolor se sustenta más en evitar y prevenir la enfermedad, el mecanismo más eficaz que tenemos hoy en día para generar en las personas un cambio de actitud, es la Educación para la Salud, en todos los ámbitos de su actuación. Dentro de ésta, la educación para la salud buco-dental, tiene una importancia trascendental (J, González, \& González García, 2019)

La Educación para la Salud como estrategia en la promoción de salud se ha constituido en una forma de enseñanza que pretende conducir al individuo y a la colectividad en un proceso de cambio de actitud y de conducta, que parte de la detección de sus necesidades y que intenta redundar en el mejoramiento de las condiciones de salud del individuo y la comunidad (Batista Castro, y otros, 2019)

Son muy importantes las actividades de Educación para la Salud como herramienta fundamental de la promoción de salud para contribuir a establecer conocimientos, actitudes y valores que ayudan al individuo y al grupo a hacer elecciones y tomar decisiones adecuadas de la salud y bienestar. Incluye la esfera afectiva, la formación de sentimientos, convicciones, valores, necesidades y hábitos.(García Heredia \& Miranda Tarragó, 2009)

Debemos encaminar nuestros esfuerzos a la prevención en todas sus vertientes como son: la educación, promoción de salud, el diagnóstico de trastornos potencialmente malignos y la detección en sus primeros estadios. Ello conlleva aplicar los tratamientos más adecuados y rehabilitar a los pacientes.

Con respecto a la educación, se deben buscar los mecanismos más efectivos para motivar adecuadamente a las personas a que se realicen el auto examen bucal y se vuelva una práctica 
habitual en las familias. Educar con el ejemplo con relación a los factores de riesgo, insistiendo en el incorrecto hábito de fumar y en la importancia de una dieta adecuada.

Entre los problemas de salud bucal detectados a través de la información obtenida sobre el auto examen bucal y factores de riesgo del cáncer bucal en la población del municipio Matanzas, se identifica desconocimiento al respecto, entre las posibles causas que dificultan la realización exitosa de las acciones educativas, está la falta de materiales educativos para realizar dichas actividades de promoción y prevención estomatológica, situación considerada por los autores como motivación para el presente estudio.

Teniendo como base lo anteriormente justificado, se ha sustentado teóricamente como problema científico:

¿Cómo contribuir a la capacitación sobre el auto examen bucal y los factores de riesgo del cáncer bucal en la población?

Para darle solución a este problema nos proponemos diseñar un medio de enseñanza que integre los elementos teóricos fundamentales acerca del auto examen bucal con los procedimientos que permiten realizar el mismo de forma correcta y cuyo método de difusión se encuentre al alcance de la población.

\section{Objetivos}

General: Implementar una Guía didáctica de apoyo al Auto examen Bucal para su uso en las actividades preventivas del cáncer bucal.

Específicos:

1. Caracterizar la población según edad y sexo.

2. Identificar el nivel de conocimiento sobre elementos de salud bucal y el auto examen bucal de la población en estudio.

3. Argumentar los fundamentos teóricos que pueden servir de base para la elaboración de la Guía Didáctica de apoyo al Auto examen bucal para su uso en las actividades preventivas del cáncer bucal.

4. Elaborar Guía Didáctica de apoyo al Auto examen bucal para su uso en las actividades preventivas del cáncer bucal.

5. Validar la Guía Didáctica de apoyo al Auto examen bucal para su uso en las actividades preventivas del cáncer bucal. 
6. Aplicar la Guía Didáctica de apoyo al Auto examen bucal para su uso en las actividades preventivas del cáncer bucal.

7. Evaluar los resultados obtenidos en la aplicación de la Guía Didáctica de apoyo al Auto examen bucal para su uso en las actividades preventivas del cáncer bucal

\section{Diseño metodológico}

\section{Tipo de estudio y diseño de la investigación.}

Se realizó una investigación de innovación tecnológica con diseño experimental, donde a partir de la realización del diagnóstico educativo se identificaron las necesidades de aprendizaje de la población de Matanzas con respecto al auto examen bucal, las autoras apoyadas en estos elementos diseñaron un medio de enseñanza. El presente producto se comenzó a aplicar en diciembre del año 2018 en la Casa de Abuelos "Antonio Dicirst Martínez", situada en la calle Río esquina Ayuntamiento, municipio Matanzas.

\section{Universo y muestra}

El universo de trabajo coincide con la muestra, que la constituyeron la totalidad de la población conformada por 47 individuos de más de 60 años de edad.

El equipo de investigadores estuvo integrado por la docente de la carrera de Estomatología que imparte la asignatura de Promoción de Salud y Prevención de afecciones estomatológicas y un colectivo de estudiantes de primer año de la carrera de Estomatología.

\section{Criterio de inclusión y de exclusión}

\section{Criterio de inclusión:}

- Capacidad psicológica para responder a las preguntas de la entrevista (sujeto mentalmente apto).

- Manifestar por escrito su consentimiento para participar en el estudio, según los criterios bioéticos.

- Ambos sexos.

\section{Criterios de Exclusión}

- Los que no cumplieron los criterios de inclusión

\section{Consideraciones bioéticas}


Se tuvo en cuenta los principios bioéticos, implícitos en todo estudio realizado con seres humanos. La información obtenida en la ejecución de la etapa diagnóstica fue consultada previamente con las personas incluidas en la investigación, las cuales confirmaron de forma voluntaria su disposición a participar, siendo informados de forma oportuna sobre las características del estudio. En la información interpersonal no se empleó lenguaje técnico, sino sencillo y comprensible para el sujeto. Se le explicó a cada paciente que la información relacionada con su identidad sería tratada de manera confidencial. Los investigadores no coaccionaron, ni influyeron para participar o mantenerse en el estudio, considerado este la máxima expresión del principio de autonomía.

\section{Operacionalización de las variables}

Definición, clasificación y operacionalización de las variables:

En cuanto al cumplimiento del primer y segundo objetivo se contemplaron variables relacionadas con edad, sexo, y sobre algunos factores relacionados con el nivel de conocimientos en cuanto a salud bucal y el auto examen bucal.

La salida del tercer objetivo de la investigación se centró en las bases teóricas de temas como: cáncer bucal, Programa de Detección del cáncer bucal y auto examen bucal. Se realizó una revisión bibliográfica mediante la consulta de bases de datos de los sistemas referativos, como medline y PubMed con la utilización de descriptores como "oral cáncer" OR "oral cáncer prevention" OR "oral cáncer programme" OR "oral cáncer early detection". Se revisó toda la bibliografía haciendo énfasis en la encontrada de los últimos cinco años.

En respuesta al cuarto y quinto objetivo se realiza el diseño del producto (medio de enseñanza) el cual es validado por expertos en la especialidad.

Para dar salida al sexto objetivo se aplica la Guía de apoyo al Auto examen bucal en las actividades preventivas del cáncer bucal en la Casa de Abuelos “Antonio Dicirst Martínez”, ubicada en el municipio Matanzas. Además, se dispone su generalización en todas las actividades de promoción y prevención realizadas por los estudiantes de la carrera de Estomatología en la comunidad.

Tabla 1. Variables sociodemográficas

\begin{tabular}{|c|l|l|l|l|}
\hline \multirow{2}{*}{ Variables } & \multirow{2}{*}{ Tipo } & \multicolumn{2}{|c|}{ Operacionalización } & \multirow{2}{*}{ Indicador } \\
\cline { 3 - 5 } & Escala & \multicolumn{1}{c|}{ Descripción } & \\
\hline \multirow{2}{*}{ Sexo } & $\begin{array}{l}\text { Cualitativa } \\
\text { nominal }\end{array}$ & $\begin{array}{l}\text { Femenino } \\
\text { Masculino }\end{array}$ & $\begin{array}{l}\text { Condición orgánica } \\
\text { que distingue al al } \\
\text { hombre de la mujer }\end{array}$ & $\begin{array}{l}\text { Frecuencias } \\
\text { absolutas y relativas }\end{array}$ \\
\hline
\end{tabular}




\begin{tabular}{|c|c|c|c|c|}
\hline Edad & $\begin{array}{l}\text { Cuantitativa } \\
\text { continua }\end{array}$ & $\begin{array}{l}\text { De } 60 \text { a } 69 \\
\text { De } 70 \text { a } 79 \\
\text { más de } 80\end{array}$ & Según años cumplidos & $\begin{array}{l}\text { Frecuencias } \\
\text { absolutas y relativas }\end{array}$ \\
\hline
\end{tabular}

Fuente: Elaboración propia.

Tabla 2. Variable nivel de conocimiento sobre salud bucal

\begin{tabular}{|c|c|c|c|c|}
\hline Variable & Dimensión & Tipo & $\begin{array}{c}\text { Operacionalización } \\
\text { Escala }\end{array}$ & Indicador \\
\hline \multirow{4}{*}{$\begin{array}{l}\text { Conocimiento } \\
\text { sobre salud } \\
\text { bucal }\end{array}$} & $\begin{array}{l}\text { Conocimientos e } \\
\text { interés sobre } \\
\text { Salud Bucal }\end{array}$ & Cualitativa & $\begin{array}{l}\mathrm{Si} \\
\text { No }\end{array}$ & $\begin{array}{l}\text { Frecuencias } \\
\text { absolutas } \\
\text { relativas }\end{array}$ \\
\hline & $\begin{array}{lr}\text { Presencia } & \mathrm{de} \\
\text { hábitos } & \text { tóxicos } \\
\text { (tabaquismo } & \mathrm{y} \\
\text { alcoholismo) } & \end{array}$ & Cualitativa & $\begin{array}{l}\mathrm{Si} \\
\text { No }\end{array}$ & $\begin{array}{l}\text { Frecuencias } \\
\text { absolutas y } \\
\text { relativas }\end{array}$ \\
\hline & $\begin{array}{l}\text { Conocimiento } \\
\text { sobre }\end{array}$ & Cualitativa & $\begin{array}{l}\mathrm{Si} \\
\text { No }\end{array}$ & $\begin{array}{l}\text { Frecuencias } \\
\text { absolutas } \\
\text { relativas }\end{array}$ \\
\hline & $\begin{array}{l}\text { Conocimiento } \\
\text { sobre consulta al } \\
\text { profesional de la } \\
\text { salud si presenta } \\
\text { alteración en } \\
\text { cavidad bucal }\end{array}$ & Cualitativa & $\begin{array}{l}\mathrm{Si} \\
\text { No }\end{array}$ & $\begin{array}{l}\text { Frecuencias } \\
\text { absolutas } \\
\text { relativas }\end{array}$ \\
\hline
\end{tabular}

Fuente: Elaboración propia.

En cuanto al cumplimiento del séptimo objetivo, se aplicó un instrumento para medir el grado de satisfacción de los participantes en el estudio después de empleado el material educativo diseñado por los autores, el cual se describe en la operacionalización de las variables.

Tabla 3. Variable evaluación de la aplicación del producto

\begin{tabular}{|c|c|c|c|c|}
\hline \multirow{2}{*}{ Variables } & \multirow{2}{*}{ Tipo } & \multicolumn{2}{|c|}{ Operacionalización } & \multirow{2}{*}{ Indicador } \\
\hline & & Escala & Descripción & \\
\hline $\begin{array}{l}\text { Valoración de la } \\
\text { aplicación del medio } \\
\text { de enseñanza }\end{array}$ & $\begin{array}{l}\text { Cualitativa } \\
\text { ordinal } \\
\text { politómica }\end{array}$ & $\begin{array}{l}\text { Positivo } \\
\text { Negativo } \\
\text { Interesante }\end{array}$ & $\begin{array}{l}\text { Según el criterio } \\
\text { de la persona } \\
\text { encuestada }\end{array}$ & $\begin{array}{l}\text { Frecuencias } \\
\text { absolutas } \\
\text { relativas }\end{array}$ \\
\hline
\end{tabular}

Fuente: Elaboración propia. 
Se aplicó la técnica evaluativa PNI (positivo, negativo e interesante) y se tomó en consideración un solo criterio de cada persona, se solicitó argumentar en caso de seleccionar negativo.

\section{Descripción general de la Guía de apoyo.}

Está conformado por:

Portada: presenta el título del producto y orientaciones generales acerca del contenido del plegable.

Página 1: Factores de riesgo del cáncer bucal y las instrucciones para realizar el procedimiento del Auto examen bucal.

Página 2, 3, 4: Pasos de cómo realizar el auto examen bucal, apoyado con imágenes sobre los diferentes pasos.

Contraportada: Señales de alarma del cáncer bucal

Las imágenes que se utilizan apoyan la descripción de los pasos a realizar en el auto examen bucal, que permiten facilitar la comprensión del texto.

Se utiliza el lenguaje materno, de fácil comprensión para todas las edades y su confección resulta económico.

Su práctica y correcta utilización facilita un aprendizaje significativo, pues demuestra visualmente los pasos a seguir en el auto examen bucal, por lo que garantiza los requerimientos mínimos para la aplicación del producto.

Procedimiento para el análisis de la información y procesamiento estadístico.

Los resultados se presentaron en cuadros y gráficos estadísticos. Se empleó una computadora Pentium IV, con ambiente de Windows XP. Los textos se procesaron en Microsoft Office Word 2007 y las tablas y gráficos se realizaron con Microsoft Office Excel 2007. A través del contraste y comparación de los resultados con la literatura científica se realizaron las interpretaciones que permitieron arribar a las conclusiones.

\section{Resultados:}

Tabla 4. Caracterización de personas según edad y sexo. Casa de abuelos Antonio Dicirst Martínez. Diciembre 2018.

\begin{tabular}{|c|c|c|c|}
\hline \multirow{2}{*}{ Edad } & & Sexo & \multirow{2}{*}{ Total } \\
\cline { 2 - 3 } & Masculino & Femenino & \multirow{2}{*}{} \\
\hline
\end{tabular}




\begin{tabular}{|l|c|c|c|c|c|c|}
\hline & No & \% & No & \% & No & \% \\
\hline 60 a 69 & 6 & 27.27 & 6 & 24.00 & 12 & 25.53 \\
\hline 70 a 79 & 13 & 59.09 & 17 & 68.00 & 30 & 63.82 \\
\hline Más de 80 & 3 & 13.63 & 2 & 8.00 & 5 & 10.63 \\
\hline Total & 22 & 46.80 & 25 & 53.19 & 47 & 100.00 \\
\hline
\end{tabular}

Fuente: Elaboración propia.

Al analizar la Tabla 1 se reflejó que el grupo etáreo que predominó fue el de 70 a 79 años $(63.82 \%)$. En cuanto a género, las féminas alcanzaron el $53.19 \%$ del total

Tabla 5. Distribución de personas según edad y conocimientos e interés sobre salud bucal. Casa de abuelos Antonio Dicirst Martínez. Diciembre 2018.

\begin{tabular}{|c|c|c|c|c|c|c|c|c|c|c|}
\hline \multirow{4}{*}{ Edad } & \multicolumn{8}{|c|}{ Salud Bucal } & \multirow{3}{*}{\multicolumn{2}{|c|}{ Total }} \\
\hline & \multicolumn{4}{|c|}{ Conocimientos } & \multicolumn{4}{|c|}{ Interés } & & \\
\hline & \multicolumn{2}{|c|}{ Sí } & \multicolumn{2}{|r|}{ No } & \multicolumn{2}{|c|}{ Sí } & \multicolumn{2}{|c|}{ No } & & \\
\hline & No & $\%$ & No & $\%$ & No & $\%$ & No & $\%$ & No & $\%$ \\
\hline 60 a 69 & 4 & 33.33 & 8 & 66.66 & 12 & 28.57 & _ & & 12 & 25.53 \\
\hline 70 a 79 & 8 & 26.66 & 22 & 73.33 & 26 & 61.90 & 4 & 80.00 & 30 & 63.82 \\
\hline Más de 80 & 1 & 20.00 & 4 & 80.00 & 4 & 9.52 & 1 & 20.00 & 5 & 10.63 \\
\hline Total & 13 & 27.65 & 34 & 72.3 & 42 & 89.36 & 5 & 10.63 & 47 & 100.00 \\
\hline
\end{tabular}

Fuente: Elaboración propia.

Al observar los resultados relacionados con los conocimientos de salud bucal en la Tabla 2, el $72.3 \%$ no refirió conocimiento sobre este tema y el $89.36 \%$ señaló interés por aprender sobre salud bucal.

Tabla 6. Distribución de personas según edad y presencia de hábitos tóxicos. Casa de abuelos Antonio Dicirst Martínez. Diciembre 2018.

\begin{tabular}{|c|c|c|c|c|c|c|c|c|c|c|}
\hline \multirow{4}{*}{ Edad } & \multicolumn{8}{|c|}{ Hábitos tóxicos } & \multirow{3}{*}{\multicolumn{2}{|c|}{ Total }} \\
\hline & \multicolumn{4}{|c|}{ Tabaquismo } & \multicolumn{4}{|c|}{ Alcoholismo } & & \\
\hline & \multicolumn{2}{|r|}{ Sí } & \multicolumn{2}{|c|}{ No } & \multicolumn{2}{|c|}{ Sí } & \multicolumn{2}{|c|}{ No } & & \\
\hline & No & $\%$ & No & $\%$ & No & $\%$ & No & $\%$ & No & $\%$ \\
\hline 60 a 69 & 5 & 35.71 & 7 & 21.21 & 1 & 25.00 & 11 & 28.94 & 12 & 25.53 \\
\hline 70 a 79 & 8 & 57.14 & 22 & 66.66 & 3 & 75.00 & 27 & 71.05 & 30 & 63.82 \\
\hline Más de 80 & 1 & 7.14 & 4 & 12.12 & - & - & - & - & 5 & 10.63 \\
\hline Total & 14 & 29.78 & 33 & 70.21 & 4 & 8.51 & 38 & 80.85 & 47 & 100.00 \\
\hline
\end{tabular}

Fuente: Elaboración propia. 
La distribución de los encuestados según presencia de hábitos tóxicos (Tabla 3), señaló que el $70.21 \%$ del total no presenta el hábito de fumar y con respecto al consumo de alcohol el $80.25 \%$ manifestó no presentarlo.

Tabla 7. Caracterización de personas según edad, conocimiento sobre auto examen bucal y conducta a seguir ante alteraciones de la cavidad bucal. Casa de abuelos “Antonio Dicirst Martínez”. Diciembre 2018.

\begin{tabular}{|c|c|c|c|c|c|c|c|c|c|c|}
\hline \multirow{3}{*}{ Edad } & \multicolumn{4}{|c|}{$\begin{array}{c}\text { Conocimiento sobre Auto } \\
\text { examen bucal }\end{array}$} & \multicolumn{4}{|c|}{$\begin{array}{c}\text { Consulta ante alteraciones } \\
\text { bucales }\end{array}$} & \multirow{2}{*}{\multicolumn{2}{|c|}{ Total }} \\
\hline & \multicolumn{2}{|c|}{ Sí } & \multicolumn{2}{|c|}{ No } & \multicolumn{2}{|c|}{ Importante } & \multicolumn{2}{|c|}{$\begin{array}{c}\text { No } \\
\text { importante }\end{array}$} & & \\
\hline & No & $\%$ & No & $\%$ & No & $\%$ & No & $\%$ & No & $\%$ \\
\hline 60 a 69 & - & - & 12 & 100.00 & 10 & 26.31 & 2 & 22.22 & 12 & 25.53 \\
\hline 70 a 79 & - & - & 30 & 100.00 & 23 & 60.52 & 7 & 77.77 & 30 & 63.82 \\
\hline Más de 80 & - & - & 5 & 100.00 & 5 & 13.15 & - & - & 5 & 10.63 \\
\hline Total & - & - & 37 & 100.00 & 38 & 80.85 & 9 & 19.14 & 47 & 100.00 \\
\hline
\end{tabular}

Fuente: Elaboración propia.

En el nivel de conocimientos sobre auto examen bucal, Tabla 4, se apreció que el $100 \%$ de los encuestados desconocen sobre el tema. El 80.85\% manifestó que es importante asistir a consulta ante la presencia de alteraciones en la cavidad bucal.

Tabla 8. Distribución de personas según edad y criterio de satisfacción del producto aplicado. Casa de abuelos Antonio Dicirst Martínez. Diciembre 2018.

\begin{tabular}{|l|c|r|c|c|c|c|}
\hline \multirow{4}{*}{ Edad } & \multicolumn{4}{|c|}{ Criterio de satisfacción } & \multicolumn{2}{c|}{ Total } \\
\cline { 2 - 7 } & \multicolumn{3}{|c|}{ Positivo } & \multicolumn{2}{c|}{ Interesante } & \multicolumn{2}{c|}{} \\
\cline { 2 - 7 } & No & $\%$ & No & $\%$ & No & $\%$ \\
\hline 60 a 69 & 12 & 29.26 & - & - & 12 & 25.53 \\
\hline 70 a 79 & 26 & 61.90 & 4 & 66.66 & 30 & 63.82 \\
\hline Más de 80 & 3 & 7.14 & 2 & 33.33 & 5 & 10.63 \\
\hline Total & $\mathbf{4 1}$ & $\mathbf{8 7 . 2 3}$ & $\mathbf{6}$ & $\mathbf{1 2 . 7 6}$ & $\mathbf{4 7}$ & $\mathbf{1 0 0 . 0 0}$ \\
\hline
\end{tabular}

Fuente: Elaboración propia.

En la Tabla 5 se evidenció la satisfacción de los implicados en la investigación con la aplicación del medio de enseñanza ya que el $87.23 \%$ lo consideró positivo y el $12.76 \%$ como interesante, sin ningún criterio negativo. 


\section{Discusión de los resultados}

La sociedad envejece rápidamente, el incremento del número de personas de más de 60años, es el factor poblacional que caracteriza los cambios demográficos actuales, junto al descenso de la natalidad. Cuba es un ejemplo de país en desarrollo con un envejecimiento importante de su población, con una expectativa de vida al nacer actual de 75 años. Lo anterior se refleja en los resultados alcanzados en la investigación, donde se destaca en la Tabla 1 que el grupo de edad más relevante fue el de 70 a 79 años de edad, las autoras consideran con respecto esto que resultan similares en cuanto a otras bibliografías consultadas, donde el grupo etáreo más significativo es el de 70 a 79 años de edad, así refiere el Dr. Alexis Peña González y colaboradores en su artículo sobre el comportamiento clínico y epidemiológico del cáncer de cavidad oral, donde se observó el grupo de 70- 79 años, con 24, para el 53,16 \%.(A, Arredondo López, \& Vila Martínez, 2006)

Asociado a este aumento de envejecimiento de la población se han presentado cambios en la expectativa de vida al nacer, conservando las mujeres una expectativa superior de alrededor de 6 años, esto apoya los resultados alcanzados en nuestro estudio donde alcanzaron el 53.19 $\%$ del total. En bibliografía consultada también coinciden autores con nuestros criterios, González Heredia (E, 2000)señala en su investigación que el comportamiento por sexo mostró predominante a las mujeres con el 60,8 \%, estos resultados pueden estar explicado por la sobre mortalidad masculina para estos grupos de edades, las mujeres viven por término medio bastante más que los hombres, (E, 2000) criterio que coincide con las autoras.

En la Tabla 2 se recopilaron los conocimientos sobre aspectos de salud bucal e interés en conocer del tema, llama la atención que el $72.3 \%$ de los encuestados tiene dificultades en cuanto a los aspectos de salud bucal y el $89.36 \%$ manifiesta interés en familiarizarse con la temática. Estos conocimientos inadecuados en los gerontes relacionados con su salud bucal coinciden con los estudios de Sáez (R, Carmona , Jiménez Quintana, \& Alfaro, 2007), Díaz (L, Ferrer González, García Díaz , \& Duarte Escalante, 2002), Hernández (A H. , 2005), Rodríguez (LO, Reyes Puig, \& Blanco Ruiz, 2007) y Núñez (L N. A., Sanjurjo Alvarez, Gonzalez Heredia , \& Ramón Rodriguez, 2006) quien citando a Sanjurjo (L N. A., y otros, 2001)aprecia visiblemente que utilizando métodos que despierten la motivación, así como actuando en lo emotivo y cognoscitivo de la población, pueden obtenerse resultados estimulantes, que transformen positivamente y proporcionen conocimientos generales sobre salud bucal.

Un estilo de vida saludable incluye evitar el tabaquismo, la obesidad y el consumo de alcohol, así como procurar una alimentación sana, realizar actividad física y recibir atención médica sistemática, en nuestro estudio, (tabla 3) los hábitos relacionados con el tabaquismo y alcoholismo reflejan que el $29.78 \%$ del total presenta el hábito de fumar y con respecto al consumo de alcohol el $8.51 \%$ lo presenta, coincidentes con resultados de varios autores como Muñoz (L, Esposo Nápoles, Rivacoba Novoa, \& Hidalgo, 2003)encontró tabaquismo en 27,5 
\% de los estudiados. Otros autores como Berenguer (M B. G., 1999) encuestó $37.8 \%$ de gerontes que practicaban hábitos tóxicos.

En Proyecciones de la Salud Pública en Cuba, Balaguer (JR, 2006) comentó que el tabaquismo es la principal causa de muerte evitable en el mundo y que se consideró la mayor epidemia del siglo XX, constituyendo un reto para la Salud Pública Mundial el lograr disminuirlo.

A pesar de la existencia en nuestro país de un programa de detección precoz del cáncer bucal, prima en la población el desconocimiento de esta enfermedad y se mantienen los factores de riesgo (M, Ordaz Gonzalez, Abreu Noroña, Romero Pérez, \& García Mena , 2013)

. Los resultados obtenidos en este estudio corroboran lo anterior por presentar al $100 \%$ de los encuestados con desconocimiento sobre el método preventivo del auto examen bucal. Algunos investigadores (M, Ordaz González, Abreu Noroña, Romero Pérez, \& García Mena, 2013) (GL \& Miranda Tarragó, 2009)expresan el poco conocimiento sobre la prevención del cáncer bucal, signos y síntomas y realización del auto examen. El auto examen se orienta fundamentalmente a detectar los llamados signos de alarma. Con respecto al conocimiento sobre signos de alarma y actitud ante su detección algunos autores expresan que los adultos manifiestan una actitud correcta al acudir a los servicios de salud correspondientes, sin embargo, un por ciento muy bajo refiere conocer los signos de alarma del cáncer bucal, resultados coincidentes con los de las autoras.

Otros autores, (N, y otros, 2012) (M, Santana Garay , González Docando, \& Borroto Alcorta, 2007) (Tarragó, 2014)en sus estudios, valoraron el desempeño de los estomatólogos con relación al auto examen bucal, y aseveran que solo algunos enseñan este procedimiento, que la mayoría no les indican la necesidad de realización del procedimiento a sus pacientes, ni colocan propaganda gráfica visible en los locales de consulta.

Lo señalado anteriormente respalda la intención del material educativo diseñado por las autoras para apoyo a las acciones preventivas del cáncer bucal, esta Guía para el procedimiento del auto examen bucal representa una respuesta rápida y científica que genera una implementación satisfactoria en el logro de acciones de prevención y control necesarios para la mejora continua de la salud en nuestro pueblo.

Los medios de enseñanza constituyen una herramienta útil para llevar a cabo los Programas de Educación para la Salud y como introducción del estudiante en la educación en el trabajo en las actividades de promoción y prevención. (F, y otros, 2019)

Su gran importancia radica en que a través de ellos pueden llegar mensajes educativos a la población de forma que ayuden en la ardua tarea de los profesionales de la Salud para lograr incorporar en los individuos, la familia y la comunidad comportamientos saludables, así 
como cambiar otros que no lo son. (L, Ramírez Ramírez, Serrano Díaz, \& Fernández Queija, 2019) (Ramos Francisco \& Calero Barreto, 2019)

Estos aportan elementos de importancia adicional permitiendo un mayor grado de reflexión y análisis de las personas participantes sobre los temas que los mismos aborden.

Todo esto se reafirma en los resultados en la tabla 5 que evidencia el nivel de satisfacción de los participantes en la investigación donde casi mayoritariamente opinaron como positiva e interesante el material educativo puesto en práctica sin ningún criterio negativo.

\section{Conclusiones.}

- Prevaleció el grupo de edad de 70 a 79 años y el sexo femenino fue el más elevado.

- Existió desconocimiento sobre auto examen bucal y bajo nivel de conocimiento en salud bucal. Se evidenció interés para aprender acerca de este tema. No es significativo el predominio de hábitos tóxicos (alcoholismo y tabaquismo).

- Los fundamentos teóricos desarrollados sobre los temas cáncer bucal, Programas de Detección del Cáncer Bucal y auto examen bucal sirvieron de base para la elaboración de la Guía Didáctica de apoyo al Auto examen bucal para su uso en las actividades preventivas del cáncer bucal.

- Se elaboró una Guía de apoyo al Auto examen bucal, validada por un comité de expertos que la consideró apropiada.

- La guía fue aplicada y evaluada su implementación, donde se demostró el nivel de satisfacción de la misma por los participantes en el estudio.

\section{Referencias bibliográficas.}

A, H. (2005). Trabajo para optar por el título de especialista de I Grado en Estomatología General Integral. Modificacion de conocimientos sobre salud bucal en adultos mayores del Consultorio 38. Policlinico "Dr. Carlos J. Finlay" . Santiago de Cuba, Cuba.

A, P. G., Arredondo López , M., \& Vila Martinez, L. (2006). Comportamiento clínico y epidemiológico del cáncer de cavidad oral. Rev Cubana Estomatolol , 43 (1).

Batista Castro, Z., Gonzalez Aguilar, V., García Barceló, M., Rodriguez Pérez, I., Miranda Tarragó, J., Chica Padilla, M., y otros. (2019). Evaluación clínico epidemiológica de trastornos bucales potencialmente malignos en adultos de Montalvo en Ambato, Ecuador. Rev Cubana Estomatol , 56 (4), 1-13. 
C, S. M. (2011). Manual de organización y procedimeintos para el control del cáncer bucal. Programa Nacional de Atención Estomatológica Integral a la Población , 204-26.

Calunga, P. C., \& Castillo A., D. (Enero de 2014). Conocimientos sobre el cáncer bucal en pacientes de Estomatología. Revista Cubana de Medicina Militar , 16 p.

Celedón, C. (2012). Reflexiones de algunos aspectos éticos de la asistencia en el paciente oncológico terminal de cabeza y cuello. Rev Otorrinolaringol Cir Cabeza Cuello , 72 (2), 7 .

E, G. H. (enero-junio de 2000). Tesis para optar por Máster en Atención Primaria. Aspectos principales de la salud bucal del adulto mayor en el consejo popular Guillermo Moncada, municipio Santiago de Cuba . Santiago de Cuba, Cuba.

Espinosa Ortiz, E. (12 de 09 de 2019). Tesis Factores de riesgo en pacientes con cáncer oral.

F, G., Dallegre , C., Morán , F., Miranda , a., López de Blanc, s., Gandolfo, M., y otros. (2019). Derecho ala sonrisa. Revista de Extension de la UNC , 9.

Garcia Heredia, G., \& Miranda Tarragó, J. (2009). Necesidades de aprendizaje relacionados con el cáncer bucal en un círcuo de abuelos de Ciudad de la Habana. Revista Cubana Estomatologia , 46 (4), 90-101.

GL, G. H., \& Miranda Tarragó , J. (2009). Necesidades de aprendizaje relacionados con el cáncer bucal en un círculo de abuelos de Cudad de la Habana. Rev Cubana Estomatol , 46 (4), 11.

Guerrero Brito, M., Perez Cabrera, D., \& Hernandez Abreu, N. (2020). Lesiones bucales premalignas en pacientes con hábito de fumar. Medicentro Electrónica , 24 (1), 159164.

I., C. V., Suarez Zafra , D., Leyva Labrador, Y., Rodriguez , M., \& Navarro Espinosa , M. (2009). Comportamiento del programa de prevención de Cancer Bucal. Rev inf cient , 61 (1), 1-10.

ID., V., Kalpidis , C., Chadiipantelis , T., \& Konstantinidis , A. (2009). Cigarette smoking associated with advanced periodontal destruction in a Greek sample population of patients with periodontal disease. J Int Acad Periodontol , 11 (4), 250-7.

J, C. G., Gonzalez, R., \& Gonzalez Garcia , X. (2019). Enfermedades bucales premalignas en adultos mayores del Policlínico Universitario Pedro Borrás astorga, Pinar del Río. Correo Científico Médico , 23 (3).

J., Q. C. (2007). Ambiente libre de humo de tabaco. Revista del Instituto Nacional de Higiene "Rafael Rangel" , 38 (1), 5. 
JR, B. C. (2006). Proyecciones de la salud pública en Cuba para el 2015. 7-61. Ciudad de la Habana: Ciencias Médicas.

L, D. d., Ferrer Gonzalez, S., García Diaz , R., \& Duarte EScalante, A. (2002). Modificaciones del conocimiento y actitudes hacia la salud bucal en adolescentes del Reparto Sueño. MEDISAN , 5 (2).

L, H. C., Ramirez Ramírez, R., Serrano Díaz, B., \& Fernandez Queija, Y. (2019). Lesiones cancerígenas y precancerosas bucales. Factores de riesgo asociados. Policlinico Bernardo Posse, año 2017. Rev Méd Electrón , 41 (3).

L, M. F., Esposo Nápoles, N., Rivacoba Novoa, E., \& Hidalgo, C. (2003). Salud bucodental en los ancianos, etapa diagnóstica.

L, N. A., Sanjurjo Alvarez, M., Berenguer Gouarnalusses, M., Gonzalez Heredia, E., Salvador , S., \& Delgado Correa, W. (2001). Modificación de conocimientos sobre salud bucal en el adulto mayor del Consejo Popular "Los Maceo".

L, N. A., Sanjurjo Alvarez, M., Gonzalez Heredia , E., \& Ramón Rodriguez, A. (2006). Intervencion educativa sobre salud bucal en gerontes institucionalizados de zona rural. Rev Cubana Estomatol , 43 (4).

LO, R. G., Reyes Puig, A., \& Blanco Ruiz, A. (2007). Instituto Superior de Ciencias Médicas de la Habana. Intervencion educativa sobre la salud bucal en un círculo de la tercera edad. La Habana del Este . Ciudad de la Habana, Cuba.

M, B. G. (1999). La salud bucodental en la tercvera edad, Instituto Superior de Ciencias Médicas. Facultad de Estomatología. MEDISAN , 3 (4), 53-56.

M, H. R., Santana Garay , J., Gonzalez Docando, Y., \& Borroto Alcorta, D. (2007). Factores de riesgo del cáncer bucal. MediCiego , 13 (1), 9.

M, M. F., Ordaz Gonzalez , A., Abreu Noroña , A., Romero Pérez, O., \& García Mena , M. (2013). Intervención educativa para la prevención del cáncer bucal en adultos mayores. Revista de Ciencias Médicas de Pinar del Río, 17 (4).

N, R. R., Alvarez Blanco, Y., Cardenas Perez, E., Cruz Betancourt, M., Morales Pardo, M., \& Cepero Franco, F. (2012). Caracterizacion de las remisiones del Programa de Detección Precoz del cáncer bucal al segundo nivel de atención en el area norte de Ciego de Avila. MediCiego , 18 (1), 14.

ND., M., Rodriguez, L., \& Garcia JM. (2009). Importancia del Programa de detección del Cáncer bucal en Cuba. Rev Cubana Cienc Méd , 7-8. 
Organization, W. H. (2008). Are de numbers of cancer cases increasing or decreasing in the World? World Health Organization , 5.

Pública, M. d. (2009). Anuario estadístico 2008. Dirección Nacional de Registros Médicos y Estadísticas de Salud, 8.

R, S. C., Carmona , M., Jimenez Quintana, Z., \& ALfaro, X. (2007). Cambios bucales en el adulto mayor. Rev Cubana Estomatol , 44 (4).

Ramos Francisco, M. Y., \& Calero Barreto, P. (enero de 2019). Intervención educativa para modificar conocimientos sobre cáncer bucal en trabajadores de fábrica de tabaco. Revista Caribeña de Ciencias Sociales .

RM, G. R., Ventura Hernandez, M., Valdivié Provance, J., \& Serrano Älvarez, C. (2014). Sonríe con Salud. Rev Cubana Estomatol , 51 (3), 250-258.

Rodriguez Ricardo, E., Santana Fernandez , K., Fong Gonzalez, Y., Rey Ferrales, Y., Jacas Gomez, M., \& Quevedo Peillón, K. (2014). Evaluación del programa de detección precoz del cáncer bucal. Revista Archivo Médico de Camagüey , 18 (6), 642-655.

Rodriguez Ricardo, E., Santana Fernandez, K., Fong Gonzalez, Y., Ferrales, R., Jacas Gomez, M., \& Quevedo Peillón, K. (s.f.). Evaluaci.

Salud, M. d. (2012). Plan Nacional para la Prevencion y Control del Cáncer 2011-2017. El Ministerio, 7.

Tarragó, M. (2014). Retos y posibilidades en la disminucion de la mortalidad por cáncer bucal. Rev Cubana Estomatol , 51 (3). 


\section{PARA CITAR EL ARTÍCULO INDEXADO.}

Garay Crespo, M. I., Rubiera Carballosa, J., González Escolarte, V., \& Rodríguez Domínguez, M. (2020). Guía didáctica de apoyo al Autoexamen Bucal. Anatomía Digital, 3(2), 49-67. https://doi.org/10.33262/anatomiadigital.v3i2.1188

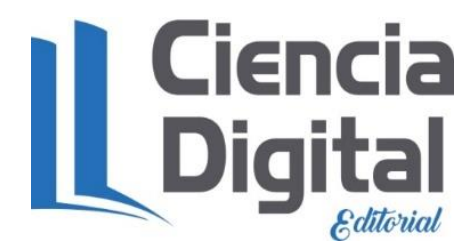

El artículo que se publica es de exclusiva responsabilidad de los autores y no necesariamente reflejan el pensamiento de la Revista Anatomía Digital.

El artículo queda en propiedad de la revista y, por tanto, su publicación parcial y/o total en otro medio tiene que ser autorizado por el director de la Revista Anatomía Digital.
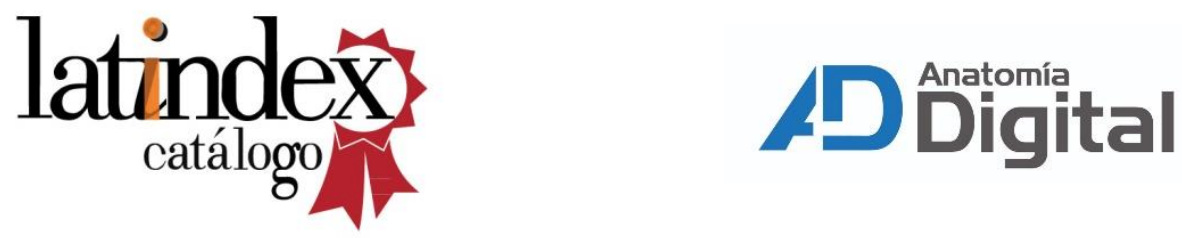